
allemande

48-2 | 2016

Les espaces publics des pays germanophones, des espaces publics transnationaux?

\title{
Deutsch auf der Bühne: Vektor für \\ Transnationalität in der öffentlichkeit?
}

\section{Aline Vennemann}

\section{(2) OpenEdition}

\section{Journals}

Édition électronique

URL : https://journals.openedition.org/allemagne/426

DOI : 10.4000 /allemagne.426

ISSN : 2605-7913

Éditeur

Société d'études allemandes

Édition imprimée

Date de publication : 28 décembre 2016

Pagination : 329-344

ISSN : 0035-0974

Référence électronique

Aline Vennemann, „Deutsch auf der Bühne: Vektor für Transnationalität in der Öffentlichkeit?", Revue d'Allemagne et des pays de langue allemande [Online], 48-2 I 2016, Online erschienen am: 28 Dezember 2017, abgerufen am 23 Mai 2021. URL: http://journals.openedition.org/allemagne/426 ; DOI: https:// doi.org/10.4000/allemagne.426 


\section{Deutsch auf der Bühne: Vektor für Transnationalität in der Öffentlichkeit?}

- Aline Vennemann*

\section{Einführung}

Dass Sprache, und insbesondere die gesprochene, performativ ist, zählt seit Austins und Searls Sprechakttheorie ${ }^{(1)}$ zu den Standards der pragmatischen Linguistik und zwar auf internationaler Ebene. Wenn die geschriebene Sprache - wie es weitere Beiträge dieses Bandes über die deutschsprachige Presse zeigen - Grenzen überschreitend auf einen sprachlich begrenzten Rezeptionskreis meinungsbildend wirkt, welchen Einfluss mag dann erst die deutschsprachige Bühne auf ihr Publikum haben? Inwiefern prägt unter den Sprechmedien die Bühnensprache die jeweiligen Landeseinwohner? Vermag sie über regionale bzw. nationale Grenzen hinaus die Öffentlichkeit (espace public) zu beeinflussen und einen Grenzen auflösenden Rezeptionsraum (espace de partage) zu schaffen? Kurz: Ist Deutsch auf der Bühne ein Vektor transnationaler Öffentlichkeitsformen oder nicht?

Unter „Vektor“ verstehe ich ganz im Sinne der Medizin nicht den Erreger, sondern den „Überträger“ einer Krankheit, also ein Phänomen, das den Zustand eines spezifischen Systems (Körpers) augenmerklich zu ändern vermag; in diesem Fall handelt es sich um den öffentlichen Raum, wobei jede Form sozialen Austauschs, die klassen- und altersübergreifend an einem frei zugänglichen Ort wirkt, als „öffentlich“ gilt.

Diese Frage stelle ich mir nicht nur als DaF-Lehrerin ${ }^{(2)}$ im täglichen Kontakt mit Schülern und Studierenden, sondern auch als leidenschaftliche Theaterbesucherin deutschsprachiger Inszenierungen im Ausland. Dabei bietet das Elsass ein äußerst

* Professeure agrégée, Lycée J.-H. Lambert (Mulhouse) / docteure en études germaniques, Université de Haute-Alsace.

1 John Langshaw Austin, How to do Things with Words, Cambridge MA, Havard University Press, 1962; John R. Searle, Speech Acts: An Essay in the Philosophy of Language, Cambridge, Cambridge University Press, 1969; John R. SeArle, Expression and Meaning. Studies in the Theory of Speech Acts, Cambridge, Cambridge University Press, 1979.

2 DaF: Deutsch als Fremdsprache. 
spannendes Untersuchungsfeld, insofern nicht nur die nationalen Bühnen wie das TNS in Straßburg oder die Filature in Mulhouse sondern auch kleinere Institutionen von Wissembourg bis St. Louis deutschsprachige Stücke zeigen. Die größeren Bühnen heben sich dadurch vom nationalen Angebot ab. Zugleich werben sie um eine doppelte Zuschauergemeinschaft, die sowohl aus Frankreich und Deutschland als auch aus der Schweiz in die Theatersäle strömt. Ein Bestreben der aktuellen Politik ist daher frankophone Stücke mit deutschen Untertiteln zu versetzen und deutschsprachige Produktionen französisch zu untertiteln. Kann man in diesem Sinn von dem Willen sprechen, einen gemeinsamen, transnationalen Zeit-Raum der Öffentlichkeit zu schaffen, der zumindest solange besteht wie die Inszenierung dauert?

Dieses Dopplungsverfahren erweist sich als besonders produktiv bei zweisprachigen, transnationalen Koproduktionen wie Je te regarde / Ich schaue dich an ${ }^{(3)}$ zwischen dem Theater Freiburg und der Filature in Mulhouse. Unter der Leitung des Schweizer Opern- und Theaterregisseurs Jarg Pataki ${ }^{(4)}$ spielt eine binationale Truppe beidseitig des Rheins ein zweisprachiges Stück mit entsprechenden Untertiteln. Wird auf der Bühne deutsch gesprochen, verdoppeln die Übertitel das Gesprochene durch französische Schriftzüge; wird jedoch auf Französisch verhandelt, sind die affichierten Texte deutschsprachig. Dies erfordert eine besondere Denk- und Lesegymnastik seitens des Publikums, das sich aus sowohl deutschsprachigen als auch frankophonen Zuschauern zusammensetzt ${ }^{(5)}$. Eine andere Resonanz erhalten internationale Produktionen wie das französischsprachige Stück La Mouette des russischen Autors Anton Tschechow, das der deutsche Kultregisseur Thomas Ostermeier am Théâtre Vidy-Lausanne im Frühjahr 2016 kreiert hat ${ }^{(6)}$ oder Falk Richters Uraufführung am TNS Je suis Fassbinder im März 2016, das mit französischen Muttersprachlern während der Proben initiiert und geschrieben wurde. Der deutsche Arbeitstext wurde dabei über Nacht ins Französische übertragen, damit ihn die frankophonen Schauspieler am Folgetag einstudieren konnten. Solche extravaganten Projekte sind jedoch abhängig von der Experimentierfreudigkeit und kulturellen Politik der Intendanz und sind in diesem Fall auf ein persönliches Anliegen des Intendanten des TNS, Stanislas Nordey, der selbst Theaterregisseur und Schauspieler ist, zurückzuführen.

Schriftsprache (Untertitel) und Bühnensprache konkurrieren dabei nicht miteinander, sondern koexistieren und befruchten sich gegenseitig, wie auch die auf der Bühne verhandelten Themen dies- und jenseits des Rheins unterschiedlich aufgefasst und ausgelegt werden. Kann man jedoch deshalb behaupten, dass die Bühnensprache Grenzen übergreifend identitätsstiftend auf eine bestimmte, in diesem Kontext transkulturelle und transnationale, Öffentlichkeit wirkt? Vermag Deutsch als Bühnensprache Einheit bzw. so etwas wie Gruppenzugehörigkeit zu stiften oder löst es eher Distanzierungsstrategien aus? Dieser Frage möchte ich hier so klar wie möglich

3 Die Uraufführung hat am 8. März 2016 in der Filature (Mulhouse) stattgefunden.

4 https://de.wikipedia.org/wiki/Jarg_Pataki

5 Das an die Inszenierung anschließende Publikumsgespräch hat gezeigt, dass sich viele Zuschauer herausgefordert fühlten und eine intellektuelle Leistung vollbringen mussten, um sowohl dem Bühnengeschehen als auch dem Text zu folgen.

6 Odéon Théâtre de L'Europe, Teatro Stabile Di Torino, La Filature - Scène Nationale Mulhouse, TapThéâtre Auditorium de Poitiers. 
nachgehen, wobei ich zuerst den Schwerpunkt auf den Normalisierungsprozess des Binnendeutschen setzen werde und anschließend anhand eines pädagogischen Projektes zwischen Hoch- und Oberschule überprüfen.

\section{Bühnendeutsch: $\mathrm{Zu}$ den Ursprüngen der deutschen Standardlautung}

Das Beispiel Theater ist nicht völlig aus der Luft gegriffen, sondern es hat mit der deutschen Sprachgeschichte zu tun. 1855 bedauerte Professor Dunker im Schleswig Holsteinischen Schulblatt, dass „Die Schriftsprache [...] dem deutschen Volke ein fremdes Idiom" sei $^{(7)}$. Dies galt umso mehr für die deutsche Lautung, die sich bis heute stärker als im französischen Sprachraum durch Varietäten und Dialekte auszeichnet. Das sogenannte „Standarddeutsch“, bezeichnet lediglich eine normierte Schriftsprache, die eine Standardlautung bis weit ins 20. Jh. ausschloss. Um der deutschen Lautung gerecht zu werden, müsste vielmehr der Plural deutsche Sprachen benutzt werden oder gar ein Neologismus wie „Varietätendeutsch“.

Hochdeutsch, wie es in den Schulen gelehrt und in den Medien gesprochen wird, ist tatsächlich eine Kunstsprache, die das plurizentrische Wesen der deutschen Sprache zugunsten einer einheitlichen Verschriftlichung (die man „Rechtschreibung“ nennt) und Aussprache nivelliert.

Stammen auch die ersten Versuche einer solchen Vereinheitlichung aus der Mitte des 18. Jh. (man denke hier z.B. an Carl Friedrich Aichingers Versuch einer teutschen Sprachlehre aus dem Jahr 1754 in Wien), so kann erst Anfang des 19. Jh. von einer ernsthaft angestrebten Standardlautung die Rede sein, und zwar durch keines geringeren Arbeit als Goethes Regeln für die Schauspieler aus dem Jahr 1803. Dieser unterstreicht dort, „das Erste und Notwendigste für den sich bildenden Schauspieler, [nämlich] daß er sich von allen Fehlern des Dialekts befreie und eine vollständige reine Aussprache zu erlangen suche. Kein Provinzialismus taug[e] auf die Bühne! Dort herrsche nur die reine deutsche Mundart, wie sie durch Geschmack, Kunst und Wissenschaft ausgebildet und verfeinert worden“(8) (Hervorhebung: A.V.). Jene „reine deutsche Mundart“, die den heutigen Verfechtern deutscher Varietäten, insbesondere im alemannischen Sprachraum, sicherlich die Haare zu Berge stehen lässt, nimmt sich fast ein Jahrhundert später der Sprachforscher Theodor Siebs an, wenn er 1898 das erste deutsche orthoepische Aussprachelexikon publiziert. Es richtet sich in erster Linie an herangehende Schauspieler ${ }^{(9)}$. Wie es das Adjektiv „orthoepisch“ suggeriert, handelt es sich um ein Handbuch, dass die „richtige“, wortwörtlich genommen, „(auf)rechte“ (gr. orthós) Aussprache (gr. epein, „sagen“) zusammenfasst. „Orthoepisches“ Sprechen ist also ein von Regionalismen „gesäubertes“, normiertes Sprechen. Nach welchen Kriterien wurde nun diese Norm festgelegt, zudem zu einem Zeitpunkt, wo die Frage der deutschen Einheit nicht nur auf territorialer, sondern auch auf sprachlicher Ebene gestellt und

7 Zitiert nach Werner Boesch, Norbert Richard Wolf, Geschichte der deutschen Sprache. Längschnitte - Zeitstufen - Linguistische Studien, Berlin, Erich Schmidt Verlag (Grundlagen der Germanistik, 47), 2004, S. 238.

8 Johann Wolfgang von Goethe, Kunsttheoretische Schriften und Übersetzungen, Bd. 17, Berliner Ausgabe, 1960, URL : http://www.zeno.org/nid/20004855973 (Dialekt, $1 \S$ ).

9 Theodor SiEBs, Deutsche Bühnenaussprache. Ergebnisse der Beratungen zur ausgleichenden Regelung der deutschen Bühnenaussprache, Albert Ahn (Hg.), Berlin/Köln/Leipzig, 1898. 
verhandelt wurde? Spiegelt nicht das Bemühen um eine Vereinheitlichung sprachlicher und kultureller Normen das Bestreben nach politischer Einheit wider? Entspricht dieses Verlangen nach einer Einheitssprache nicht dem Glauben, dass das Aufbrechen der Isoglossen, also dialektaler Grenzen, auch politische Auswirkungen hat?

Diese Hypothese mag tatsächlich dem Handeln der deutschen Klassik zu Grunde liegen oder zumindest das politische Einheitsbestreben der Spätromantik gefördert haben. Wie Goethe orientiert sich auch Siebs an den Idealen der Klassik. Wo höre man gutes Deutsch, wenn nicht auf der Bühne der Klassiker, die Werke wie Lessings Nathan oder Goethes Iphigenie zeigen, welche doch ein „reines Sprechen“ verlangen, „das überall als vorbildlich empfunden werden kann und über dem Landschaftlichen steht “(10)? Siebs lehnte jedoch die Erstellung einer Norm durch „Schaffung neuer Ausspracheregeln“ ab. Ganz im Gegenteil war er auf die „Feststellung des bestehenden Gebrauches" bedacht ${ }^{(11)}$. Er hausierte deshalb an vielen Bühnen Deutschlands, wo er die phonetischen Eigenheiten sorgfältig notierte. Um die phonetischen Gemeinsamkeiten herauszukristallisieren und offiziell handfest $\mathrm{zu}$ machen, versammelte Siebs im Anschluss an seine Beobachtungen eine Germanistenkommission, die aus drei Theaterintendanten (also Pragmatikern) und drei Linguisten (sprich Theoretikern) bestand. Das Regelwerk wurde schnell zum Standardwerk und bleibt bis heute eine unumstrittene Referenz für jeden Schauspieler und Nachrichtensprecher.

Illustration 1: Deutsche Bühnenaussprache

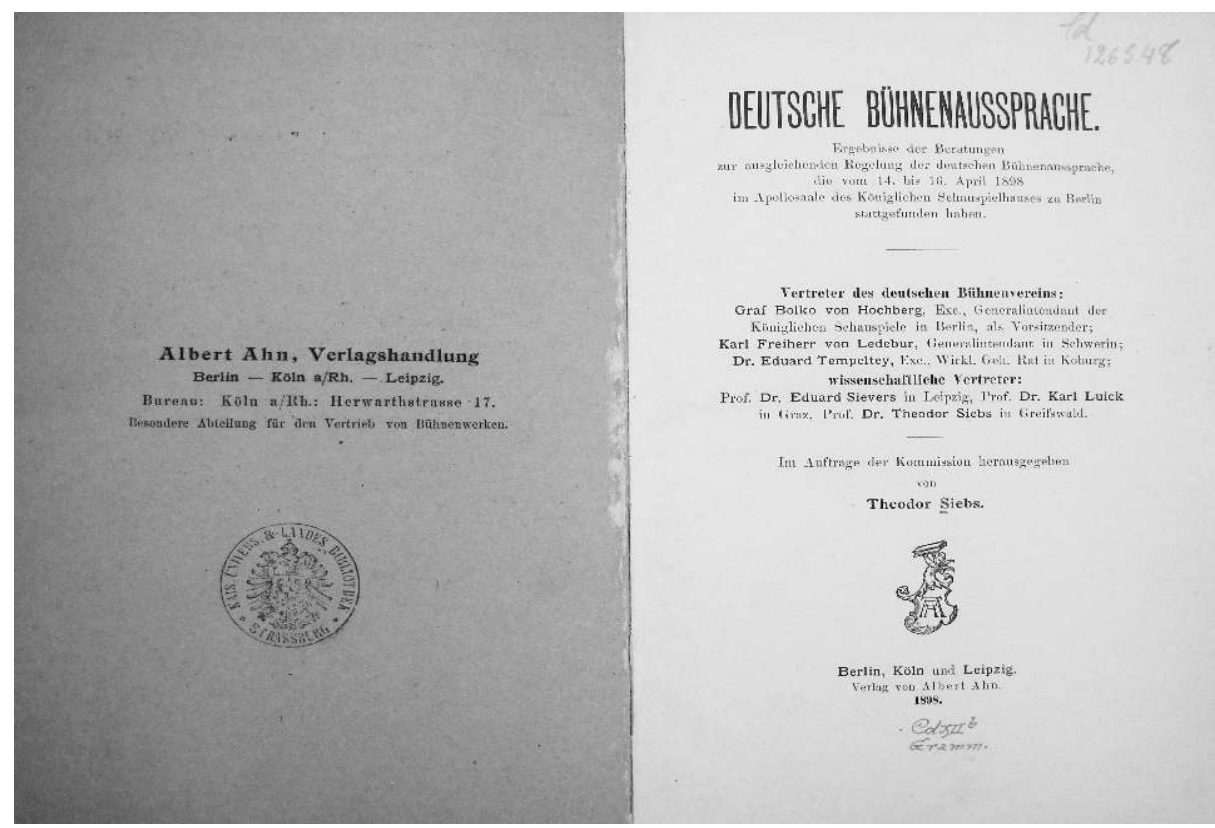

() BNU 2016

10 Vgl. „Einleitung. Begriff der deutschen Hochsprache“, in: Theodor SiEBs, Deutsche Hochsprache. Bühnenaussprache, hg. von Helmut de Boor und Paul Diels, Berlin, Walter de Gruyter \& Co, 1961 (18. Aufl.), S. 1.

11 Ebd., S. 4. 
Die hier verwendete 18. Auflage zeugt von diesem Erfolg: Sie trägt nicht mehr den ursprünglichen Titel Deutsche Bühnenaussprache, der die Aussprachenorm auf die Bühne beschränkte, sondern sie weist auf den erweiterten Resonanzraum der Studie hin, die schlichtweg zum Regelwerk der „Deutsche[n] Hochsprache“ promoviert ${ }^{(12)}$. Ein handgeklebter Einschub macht den Leser aufmerksam auf die geplante „Herstellung einer Schallplatte, die die wichtigsten Ausspracheregeln des Siebs zusammen mit einer Reihe prägnanter Beispiele“ enthalte; eine Ankündigung, die uns heute schmunzeln lässt, damals wohl aber als innovativ galt. Sie zeugt von der Modernität und dem Beliebtheitsgrad des Siebschen Standardwerks.

Doch was hat nun diese linguistische Errungenschaft mit dem Thema der „Transnationalität“ des Deutschen zu tun? Es sollen hier zwei Zitate angeführt werden, die die Eigenheit des Deutschen aus einer diachronischen Perspektive beleuchten. In seinem Monumentalwerk Der Renner beschreibt Hugo von Trimberg (um 1230 geb. - nach 1313 gest.) die deutsche Sprache in ihrer Vielfältigkeit. Sein Text spricht von vielerlei Sprachen („von manigerleie sprâche“):

„Swer tiutsche wil eben tihten,

Der muoz sîn herze rihten

Ûf manigerleie sprâche:

Swer wênt daz die Âche

Reden als die von Franken,

Dem süln die miuse danken.

Ein ieglich lant hât sînen site,

Der sînem lantvolke volget mite.

An sprâche, an mâze und an gewande

Ist unterscheiden lant von lande.

Der werlde dinc stêt über al

An sprâche, an mâze, an wâge, an zal.
Swâben ir wörter spalten,

Die Franken ein teil si valtent,

Die Beier si zezerrent,

Die Düringe si ûf sperrent,

Die Sahsen si bezückent,

Die Rînliute si verdrückent,

Die Wetereiber si würgent,

Die Mîsener si vol schürgent,

Egerlant si swenkent,

Oesterrîche si schrenkent,

Stîrlant si baz lenkent,

Kernde ein teil si senkent ${ }^{\text {“(13). }}$.

Der damalige rector scholarum aus Bamberg gibt in diesem wortgewaltigen didaktischen Gedicht (bestehend aus immerhin 25000 Paarreimen) dem nicht des Latein mächtigen Leser zu verstehen, dass es ebenso wenig eine gleichmäßige Lautung des Deutschen gebe wie gleiche Maße oder Sitten (siehe Fettgedrucktes). Der Renner galt im Spätmittelalter (1250 bis etwa 1500) als Standardwerk des damaligen Wissenstands über Literatur, Musik, Astronomie, Physik und Medizin, das den Leser zur Kenntnis des Allmächtigen führen sollte. Trimbergs sehr anschauliches Porträt der deutschen Mundarten ist für unsere Zwecke bedeutend, insofern diese frühe Bestandsaufnahme durch ein didaktisches Schriftwerk vermittelt wurde. Wenn nun Werner Boesch und Norbert Wolf rund 750 Jahre später (im Jahre 2004) schreiben:

„Deutsch ist demzufolge ein Varietätenspektrum - von der Schriftsprache und Standardsprache bis zu dialektnahen Schattierungen - frei verfügbar im Prinzip, gelöst von fixierten Herkunftszuschreibungen. Muttersprachliche ,Mehrsprachigkeit' als mögliches Modell für viele, daneben aber auch Umbau, Abbau, Abwahl von Varietäten“(14).

12 Th. Siebs, Deutsche Hochsprache (Anm. 10).

13 Hugo von Trimberg, Der Renner, hg. von Gustav Ehrismann, 4 Bde., Stuttgart, 1908-11 (Deutsche Neudrucke. Reihe: Texte des Mittelalters), V. 22253-22276.

14 Boesch/Wolf, Geschichte der Sprache (Anm. 7), S. 228. 
...so könnte man von einer Bekräftigung der mittelalterlichen Darstellung sprechen. Auch Theodor Siebs hat sich mit diesem Varietäten-Spektrum auseinandergesetzt, allerdings ohne es zu bewerten. Sein Ziel bestand nämlich darin, eine gleichmäßige Lautung aufzustellen, die sich am Bühnendeutsch orientieren sollte - also einer bestimmten Ausspracheform, die von einem möglichst breitgefächerten Publikum verstanden würde. Auf diese Weise hoffte er der schriftlichen Norm eine Standardsprache fürs Ohr entgegenzuhalten.

\section{Von Schrift zu Laut: Die Mediatisierung des Deutschen}

„Wenn mitten in einer tragischen Rede sich ein Provinzialismus eindrängt, so wird die schönste Dichtung verunstaltet und das Gehör des Zuschauers beleidigt,“ schreibt Goethe zu Beginn der Regeln für die Schauspieler ${ }^{(15)}$. Er hatte schon damals den Anspruch, einem Bildungsbürger bzw. gebildeten Publikum zu dienen.

Die Sprache der Klassik (1786-1832) verlangte von der Bühne kein Abbild der Realität, sondern eine Harmonie aller das menschliche Leben prägenden Kräfte; die Darstellung sollte ethische, ästhetische oder politische Ideale für einen Moment möglich machen oder zumindest möglich erscheinen lassen. Dem Menschheitsideal entspricht daher auch ein sprachliches Ideal, oder besser gesagt eine Idealsprache, die Goethe in seinen Regeln skizziert. Hatte Siebs durch seine Bühnenaussprache die Bühne zur „Lehrmeisterin Deutschlands“ ernannt, so unterstreichen die Verleger der 18. Auflage die „spracherzieherische Kraft“ des „,von der Bühne erklingende[n] Wort[es]“(16). Dort heißt es unverhohlen: „Vorbildliche Sprache auf der Bühne wird immer ein Maßstab der sprachlichen Kultur eines Volkes sein“ sowie „Die ,reine' Sprache der Bühne ist nicht nur ein praktisches oder technisches Anliegen der ,Fernwirkung' oder der Deutlichkeit, sie ist eine Frage der geistigen Kultur“(17).

Nähert sich auch im Gegenwartstheater die Bühnensprache immer mehr der Alltagssprache - insbesondere in zeitgenössischen, sogenannten postmodernen und postdramatischen Stücken - so tut sie dies innerhalb eines zeitlich, räumlich und sprachlich gesetzten Rahmens, der die zur Schau gestellten Sprechakte klar normiert. Bleibt der Ausruf „Scheiße“ weiterhin ein Missgriff in der Umgangssprache, erhält er auf der Bühne eine andere Funktion: Dort wird er nicht nur absichtlich gebraucht und ist Teil einer Replik, sondern er steht für einen Gemütszustand oder versinnbildlicht eine Gesellschaftsschicht.

Nicht anders verhält es sich mit den heutigen Medien (insbesondere dem Radio, das ja die visuelle Ebene nicht bedienen kann), die den Zuhörern eine besondere Sprachproduktion anbieten, eben eine „Standardsprache fürs Ohr“, möglichst homogen, ohne dialektale Tönung, in einem neutralen bis gehobenen Register und grammatikalisch sowie phonetisch einwandfrei. Exemplarisch dafür sind z.B. die Nachrichtensprecher, deren Duktus bisweilen unnatürlich wirkt ${ }^{(18)}$.

15 Goethe, Kunsttheoretische Schriften und Übersetzungen (Anm. 8), $\$ 1$.

16 Th. Siebs, Deutsche Hochsprache (Anm. 10), S. 11.

17 Ebd.

18 Anders verhält es sich in der deutschsprachigen Schweiz, wo lokale Sender durchaus in der gängigen Mundart sprechen oder in Österreich, wo die Sprecher vom „Deutschlanddeutschen“ (wie es 
Obwohl Theodor Siebs durch den ursprünglichen Titel betonte, dass sein Anliegen nicht darin bestünde, eine allgemeingültige Aussprachenorm im Alltagsleben zu bestimmen, sondern eine verbindliche Norm für das Bühnendeutsch, so hat sein Regelwerk eine breitere Wirkung erzielt, da sie den Forderungen aus dem Bildungswesen nahe kam:

„Alle öffentliche Rede, nicht nur der kunstgemäße Vortrag von Dichtung, auch die gebildete Rede auf der Kanzel, dem Katheder, dem Rednerpult und im Rundfunk, bedarf einer Pflege der Aussprache und damit eines Anhaltes an bestimmte Regeln“(19).

Gleichzeitig machten sich immer mehr Stimmen hörbar, die eine Ausweitung des initialen Geltungsbereichs der Bühne verlangten, der 1898 vom Deutschen Bühnenverein in einer Generalversammlung institutionell abgesichert wurde ${ }^{(20)}$. Tatsächlich forderten die Lehrkräfte seit einiger Zeit eine klare Regelung der Sprachproduktion, die als „lautrein“ bezeichnet würde. Schnell hat sein Werk daher die anderen Medien erobert (wie das Radio und den Film...) ${ }^{(21)}$. So rechtfertigt der Germanist bereits 1922 den Titelwechsel mit den einleitenden Worten:

„Durch lange sorgfältige Pflege hat sich auf der Bühne eine besonders reine Aussprache des Deutschen herausgebildet. Die Forderung, daß hier die Werke in einheitlicher Form dargestellt werden, und die Wechselwirkung der verschiedenen Theater aufeinander haben schon seit langer Zeit dazu geführt, daß die Aussprache der Bühne fester geregelt ward als diejenige aller anderen Kreise.

Während nirgends im deutschen Sprachgebiete eine mustergültig zu nennende Aussprache herrscht, bietet uns die deutsche Bühnenaussprache - wenn wir von ihrer vor allem auf die Deutlichkeit und starke Affekte berechneten Eigenart absehen - eine Richtschnur, die in der Wissenschaft und Kunst anerkannt und auch für andere Gebiete deutscher Sprachpflege, namentlich durch die Schule nutzbar zu machen ist. - Die deutsche Bühnenaussprache kann in diesem Sinne als deutsche Hochsprache bezeichnet werden “(22).

Es geht also indirekt um eine Spracherziehung, die nicht nur das geschriebene Wort (also Wortwahl, Syntax, Rechtschreibung, usw.), sondern auch das gesprochene betrifft - und dies im Namen einer wünschenswerten Vereinheitlichung, die regionale Spaltungen und Differenzen (von den Mundarten angefangen) überwindet.

Wenn dieses Beispiel zwar noch keinen Beweis für „Transnationalität“ liefert, so bezeugt es dennoch die Anfänge einer gewissen „Transregionalität“, oder „Überregionalität“, innerhalb eines kulturpolitischen Entwurfes des deutschen Raumes, der sich als geschlossener Sprachkörper (Reich) auch seinen Nachbarländern

dort bisweilen heißt) in den Dialekt verfallen. Allerdings sind nationale Programme und Nachrichten allgemein im Standard. Mehr dazu: http://derstandard.at/2000032954669/Oesterreichisches-Deutsch-Im-ORF-soll-es-auch-ein-bisschen-serbeln; http://orf.at/stories/2330933/2329827/; http://www.oedeutsch.at/ (abgerufen: 11.05.16).

19 Th. Siebs, Deutsche Hochsprache (Anm. 10), S. 2.

20 Der Deutsche Bühnenverein hatte im Anschluss an die Kommission die „Regeln für die Bühne als Richtschnur für die deutsche Aussprache“ empfohlen. Vgl. Deutsches Aussprachewörterbuch, „Geschichte, Grundsätze und Methoden der Ausspracheregelung in Deutschland - Die Standardsprache in Deutschland“, hg. von Eva-Maria Krech, Eberhard Stock, Ursula Hirschfeld, Lutz Christian Anders, Berlin, Walter de Gruyter, 2010, S. 10.

21 Th. Siebs, Deutsche Hochsprache (Anm. 10), S. 2-3.

22 Ebd., S. 3. 
gegenüberzustellen vermochte ${ }^{(23)}$. Der Nationalisierungsprozess ist damit auch ein Prozess des Ausschlusses der anderen (Nichtsprecher). Man kann sich daher fragen, inwieweit solch eine Maßnahme identitätsstiftend wirkt, und dies nicht im exklusiven, sondern im inklusiven Sinn.

Der am Norddeutschen und an der Schriftsprache orientierte Aussprachekatalog sollte vorerst die innerdeutschen Varianten im Sprachgebrauch, dialektale Färbungen und Varietäten des Deutschen nivellieren, die damals (bisweilen noch heute) ein Obstakel für Kommunikation und Austausch im öffentlichen Raum bild(et)en. Die sprachliche Nivellierung hatte wohl auch eine kommunikationsfördernde Funktion, die durchaus mit der klassischen Vorstellung einer ethisch-ästhetischen Erziehung durch Kunst und Theater vergleichbar ist ${ }^{(24)}$. Geht man von den bis heute im deutschen Sprachraum herrschenden Isoglossen aus, kann man deshalb sagen, dass Goethes und Siebs Bemühungen um eine "gute“ und „richtige“ Aussprache eine Art „inszeniertes“ Deutsch geradezu promoviert haben. Darüber hinaus hat sich dessen performative Wirkung rasch bemerkbar gemacht; denn knapp zwanzig Jahre später ist das norddeutsche Regelwerk zum Standardwerk der deutschen Aussprache geworden, das bis heute nicht nur ein Wegweiser für die Kunst, sondern auch für Wissenschaft, Politik und Bildungswesen geblieben ist ${ }^{(25)}$, und als Referenz für Deutschlehrer im Ausland gilt.

Dies zeigt ein genauer Blick auf die Druckgeschichte des Werks, auf die ich hier jedoch nur begrenzt eingehen kann. Das Werk wurde zwischen 1901 und 1905 in einer Kursfassung neu aufgelegt ${ }^{(26)}$. 1907 wird es auf seine Stichhaltigkeit hin überprüft. Die Forderung stammt von der Genossenschaft Deutscher Bühnenangehöriger: Fragebögen werden an rund 200 deutsche Bühnen versandt; nach Eingang der Antworten wird eine Prüfungskommission in den Kammerspielen des Deutschen Theaters im März 1908 einberufen, die sowohl aus Bühnenkünstlern, Vortragslehrern, Wissenschaftlern und Siebs und Sievers bestand: „Das Werk ging aus dieser Überprüfung so gut wie unangetastet hervor; nur in wenigen Punkten wurde es geändert oder eine Formulierung schärfer gefaßt“(27). Die 4. Auflage erscheint mit einem Aussprachewörterbuch und wird vom Patronat des Deutschen Bühnenvereins sowie der Genossenschaft Deutscher

23 Der im Zweiten Reich begonnene Nationalisierungsprozess durch Sprachvereinheitlichung akzentuierte sich unter der Ägide des Nationalsozialismus, der den „nordischen Menschen“ nicht nur durch Sprache erzeugen wollte.

24 Gemeint sind die Bestrebungen Schillers mit seinen Schriften über die Erziehung des Menschen durch Ästhetik und insbesondere das klassische Theater. Vgl. Friedrich von Schiller, Lettres sur l'éducation esthétique de l'homme / Briefe über die ästhetische Erziehung des Menschen, Paris, Aubier, 2014. In dieser bilingualen Veröffentlichung stellt Schiller seine Vorstellung des Schönen vor sowie seine Gedanken über eine mögliche Lösung der politischen Unruhen, die die Französische Revolution hervorgerufen hatte. Seine Spekulationen über die Ästhetik sollen nicht nur der Staatsreform dienen, sondern auch zum allgemeinen Glück der Menschheit führen.

25 „Allein das Werk hat sich durchgesetzt und in all seinen Teilen als sehr standfest gezeigt. Es wurde nicht nur bei der Bühne und in der Wissenschaft bald als das maßgebliche Werk für alle Fragen der Aussprache anerkannt, es wirkte über den engen Kreis seines Ursprungs hinaus in die Schule und in alle bewußte Sprachpflege und Sprecherziehung hinein." Th. SiEBs, Deutsche Hochsprache (Anm. 10), S. 20.

26 Vgl. Grundzüge der deutschen Bühnenaussprache.

27 Th. Siebs, Deutsche Hochsprache (Anm. 10), S. 21. 
Bühnenangehöriger betrieben. Seitdem hat es bis ins letzte Viertel des 20. Jh. keine wirkliche Neubearbeitung gegeben. Erst nach dem Tode Theodor Siebs 1941 sowie nach dem Ende des 2. Weltkrieges initiiert der Deutsche Verein für Sprechkunde und Sprecherziehung eine Aufarbeitung und Modernisierung des Werkes. Allerdings erfolglos - denn bis 1969 bleibt die „Regelung der alten Bühnenaussprache im wesentlichen unverändert beibehalten“(28).

Nun stellt sich die Frage, ob und inwiefern solche Aussprachreformen einen homogenen interregionalen Rezeptionsraum schaffen können, der über nationale Grenzen hinaus identitätsstiftend wirkt. War die Normalisierung der deutschen Sprache im ausgehenden 19. Jh. Teil eines innerpolitischen Nationalisierungsbestrebens ${ }^{(29)}$ (ein Land = eine Sprache), das insbesondere von den öffentlichen Erziehungsinstanzen wie der Schule (oder eben dem Theater) getragen und gefördert wurde, und somit an die Frage des „Deutschen“ gekoppelt war, so hat sie im Laufe des 20. Jh., im Kontext der zwei Weltkriege und der anschließenden Internationalisierung, wohl andere Dimensionen gewonnen, wie z.B. die eines verstärkten Sendungsbewusstseins von Sprache und Kultur. So erfüllt die Lehre einer normierten Sprache im Ausland durchaus die Funktion, in sprachlich festgelegter Form die gesellschaftlichen Gepflogenheiten und Kommunikationsformen zu vermitteln. Dabei werden dann tatsächlich die Landesgrenzen überschritten und ein weiterer, diesmal transnationaler Rezeptionsraum geöffnet.

\section{Probebeispiel Schule: zwischen Theaterpraxis und Gedenken - zweisprachige Schüler auf der Bühne ${ }^{(30)}$}

„Besonders wichtig ist die ,Hochsprache` im Unterricht für Ausländer. Wer [die deutsche] Sprache erlernt, muß von Anfang an zu einer reinen Aussprache erzogen werden“, heißt es in der 18. Auflage des Siebschen Werkes (1961):

„Der Lehrer, der Ausländer zu unterrichten hat, muß sich in seiner eigenen Aussprache vor allem gewissenhaft an die Regeln der Hochsprache halten. Denn hier ist die vernachlässigte oder mundartlich gefärbte Aussprache des Lehrers besonders verhängnisvoll, da sie unkontrolliert als Vorbild hingenommen und nachgeahmt wird. Der lernende Ausländer wird sich aber auch selber an den Normen [der] ,Hochsprache“ und ihren phonetischen Hinweisen bilden und kontrollieren müssen“(31).

Der Alltag eines Lehrers zeigt jedoch, dass die Forderung nach der sog. „Hochsprache" nicht nur Teil der Fremdsprachenausbildung ist, sondern von den Lernenden erwartet wird. Dialekte verunsichern und Varietäten stoßen manchmal auf Unverständnis - wie es nun am Beispiel eines viermonatigen mehrsprachigen Schulprojektes offengelegt werden soll.

28 Vgl. 15. Auflage 1957.

29 Und zwar des Zweiten Kaiserreiches.

30 In diesem letzten Teil meiner Ausführungen versuche ich mit theoretischer Distanz meine eigene Arbeit betrachten. Das Projekt wird in ähnlicher Variante weitergeführt, so dass der Arbeitsprozess im Fokus steht, der akute Überlegungen widerspiegelt und noch keine endgültigen Ergebnisse zu Rede stellt.

31 Th. Siebs, Deutsche Hochsprache (Anm. 10), S. 10. 
Deutschlernende aus unterschiedlichen Oberschulen, darunter die AbiBac-Schüler ${ }^{(32)}$ des Lycée Jean-Henri Lambert, sowie der oberrheinischen Universität in Mulhouse haben sich mit teils unveröffentlichten Schriften aus dem ersten Weltkrieg auseinandergesetzt ${ }^{(33)}$. Darunter befanden sich sowohl Bänkelsänge als auch Wiegenlieder, Briefe, Novellen, Gedichte und Erinnerungen von hauptsächlich elsässischen Soldaten an der „Ostfront“ - aus deutscher Sicht war es die „Westfront“ - oder von Zivilisten, die die Erlebnisse aus dem Krieg eindrucksvoll schildern. Da das Elsass im ausgehenden 19. Jh. erneut Teil Deutschlands wurde, sind die meisten Texte aus damaliger Zeit in deutscher Sprache verfasst ${ }^{(34)}$.

Ziel des Kooperationsprojektes war es, diese Zeitdokumente auf unterschiedlichste Weise zum Sprechen zu bringen, d.h. ihnen Stimme zu verleihen, sie facettenreich $\mathrm{zu}$ vertonen und anschließend aufzuführen. Ein mehrsprachiges Lehrerkollektiv, das sich aus Muttersprachlern unterschiedlicher Varietäten des Deutschen (Österreichisches Deutsch, Elsässisch und Norddeutsch bzw. „hochdeutsche“ Standardlautung) sowie aus Germanisten französischen Ursprungs zusammensetzte, hat dabei die Lernenden im Alter von 14 bis etwa 24 Jahren allwöchentlich betreut. Das Sprachniveau der Schüler erstreckte sich vom gelegentlichen B1-Sprecher bis hin zum komplexere Strukturen beherrschenden C2-Sprecher. Außerdem gab es einige

Illustration 2: Selbstentworfener Flyer der Studierenden der Licence 1 allemand 2014-15 für den 15. Mai (Es handelt sich um einen Kriegshelm aus dem Privatbesitz einer Studierenden, deren Großvater im Stellungskrieg im Einsatz war.)

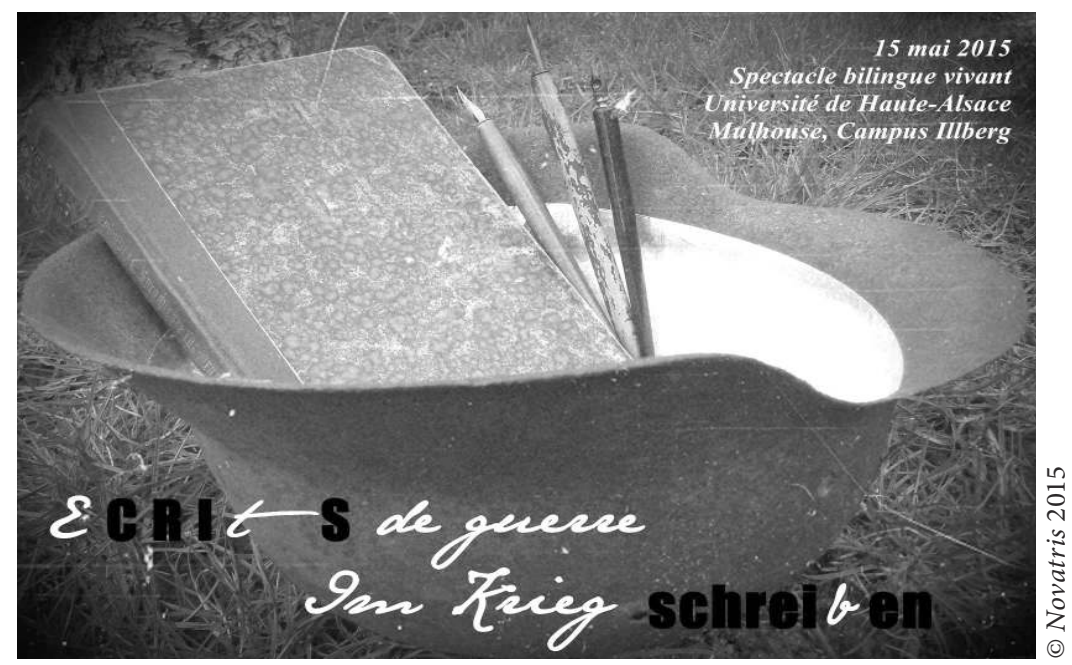

32 „Abi-Bac“ bezeichnet eine bilinguale Oberstufe, am Ende derer die Oberschüler sowohl das deutsche Abitur als auch das französische Baccalauréat absolvieren. URL: http://www.education.gouv.fr/ cid20998/1-abibac.html

33 Die Texte stammten aus dem Privatbesitz des lokalen Künstlers und Lieddichters Daniel Muringer und wurden in gemeinsamer Absprache je nach Altersgruppe und Bildungsstand ausgewählt.

34 Die meisten Elsässer haben tatsächlich auf Deutsch geschrieben; allerdings gab es auch viele, die sich des Französischen bedienten. Neben den elsässischen Egodokumenten wurden daher auch Lieder und Gedichte von Deutschen und Franzosen behandelt. 
Muttersprachler des alemannischen und elsässischen Sprachraumes. Umgangssprache und Lehrsprache war daher Hochdeutsch. Auf disziplinärer Ebene siedelte das Projekt zwischen Geschichte, Landeskunde, Deutschunterricht und Langues et Cultures Régionales (LCR) an. Parallel dazu verlief ein Übersetzungsseminar (Version/DeutschFranzösisch) für Bachelorstudierende, deren Ergebnisse am 15. Mai 2015 durch ihre Kommilitonen im Audimax der Universität aufgeführt wurden.

Obwohl das Thema die Darsteller direkt betraf, insofern die vier ${ }^{(35)}$ kooperierenden Strukturen aus dem Umkreis von Mulhouse stammten, und von lokaler Bedeutung war durch die Nähe zum Hartmannsweilerkopf - einem der Hauptschauplätze des Stellungskrieges an der Westfront, wo noch heute das Labyrinth der Gräben ungeschützt zu sehen ist ${ }^{(36)}$, und der im elsässischen Familiengedächtnis tief verankert ist - stießen meine Kollegin ${ }^{(37)}$ und ich immer wieder auf Widerstände aller Art:

- Widerstand gegen das ungewöhnliche Arbeitsmaterial oder die ungewohnte Arbeitsweise (singen, musizieren, deklamieren, darstellen, szenischen Lesen, usw.), - aber auch Ablehnung der Mundarten: alles, was von der bekannten Schulnorm („Hochdeutsch“) abwich, führte bei einigen Lernenden zu Unverständnis und blockierte sie bisweilen.

Die ungewisse und oftmals doch ungewohnte Lautung der elsässischen oder badischen Mundart sowie die variable Rechtschreibung der ausgewählten Egodokumente, Textfragmente und Lieder verunsicherten sichtlich Schüler und Studierende, die es gewohnt waren, in hochdeutschen Aussprache- und Schreibnormen zu arbeiten. Sie wirkten auf den ersten Blick befremdlich. Hier sei angemerkt, dass es für viele Lernende der erste „mündliche“ und schriftliche Kontakt mit der elsässischen Varietät war. Jedoch bereits mit dem Elsässisch vertraute Sprecher (die aus dem Norden, Süden und der Mitte des Elsass stammten) blühten sichtlich auf und offenbarten ihren Mitschülern/Kommilitonen eine ganz andere, oftmals intime, Seite. Spannend war ebenfalls der dialektale Austausch zwischen Sprechern aus der alemannischen Schweiz, dem Sundgau oder dem Niederelsass, die die Texte nun von einem Dialekt in den ihrigen übersetzten oder sich den Dialekt des Autors aneigneten. Bezüglich der eingangs gestellten Frage, kann man also sagen, dass der Umgang mit der ungewohnten deutschen Sprachvarietät parallel zur gewohnten Hochdeutschen sowohl identitätsstiftend wirkte als auch Abgrenzungsprozesse auslöste.

Mit der Zeit gewöhnten sich die meisten nicht nur an die Sprache des vergangenen Jahrhunderts, sondern sie eigneten sich die Texte an, und zwar individuell sowie kollektiv. Die Aneignung verlief einerseits über das allmähliche Begreifen der angerissenen Konzepte, andrerseits über die Verkörperung (das Nachspielen und Empfinden) der geschilderten Szenen und Emotionen, sowie über das Sprechen selbst, d.h. über die Vergegenwärtigung der Texte durch Aussprache und Spiel. Die persönliche Aneignung und kollektive künstlerische Umsetzung erfolgte daher nicht nur unter

35 Neben dem Lycée J.-H. Lambert und der Université de Haute-Alsace nahmen die zwei Gymnasien aus Guebwiller, das Lycée Kastler und das Lycée Deck, an der Veranstaltung teil. Im Rahmen dieser Studie beziehe ich mich jedoch auf meine persönlichen Erfahrungen mit den Schülern und Studierenden.

36 Siehe: www.memorial-hwk.eu; www.ahwk.fr; www.hartmannswillerkopf.e-monsite.com

37 Nina Kulovics, ÖAD-Lektorin an der Université de Haute-Alsace; seit September 2016, Doktorandin bei Novatris. Wir arbeiten seit 2014 im Teamteaching. 
der Anleitung der Lehrkräfte, sondern wurde durch einen lokalen Musiker und Dichter (namens Daniel Muringer) unterstützt, der des Elsässischen mächtig war und für die jungen Akteure Partituren schrieb.

Auf diese Weise haben sich die Spieler (die zeitweise selbst Zuschauer waren) mit einem komplexen Kapitel der lokalen Geschichte auseinandergesetzt, die sich in einen ihnen zuvor bekannten Kontext schrieb. Die historische Aufarbeitung haben sie aber nicht in der Muttersprache (Französisch), sondern in einer Fremdsprache (Hochdeutsch bzw. „Varietätendeutsch“) geleistet, die ihnen neue Perspektiven (mögliche Blicke auf den Frontenkrieg) erschlossen hat. Zudem hat sich durch konkrete Artikulierung der Kriegsschriften eine andere Wahrnehmung der fernen Epoche - des Krieges - ergeben. Nicht nur das Publikum, sondern auch die Spieler bzw. Sprecher selbst waren sichtlich ergriffen.

Dabei spielte der Aufführungsort selbstverständlich eine wichtige Rolle: Wir hatten einen Hörsaal ausgewählt, der über eine kleine Bühne verfügt. Wissenschaft, Kunst und Pädagogik waren auf diese Weise gleichsam vertreten. Ferner evoziert die Aufführungssituation den Ausgangspunkt von Theodor Siebs: Ein Wissenschaftler nimmt an einer künstlerischen Darbietung teil, um daraus einen pädagogischen Gewinn zu ziehen... offen bleibt dabei, ob der Linguist tatsächlich Freude an der Aussprache des Deutschen als Fremdsprache in seiner Dialekt- und Aussprachevielfalt auf der improvisierten Bühne der oberrheinischen Universität gehabt hätte.

Illustration 3: Endszene der Veranstaltung am 15. Mai 2015 im Amphi Weiss, UHA/Illberg

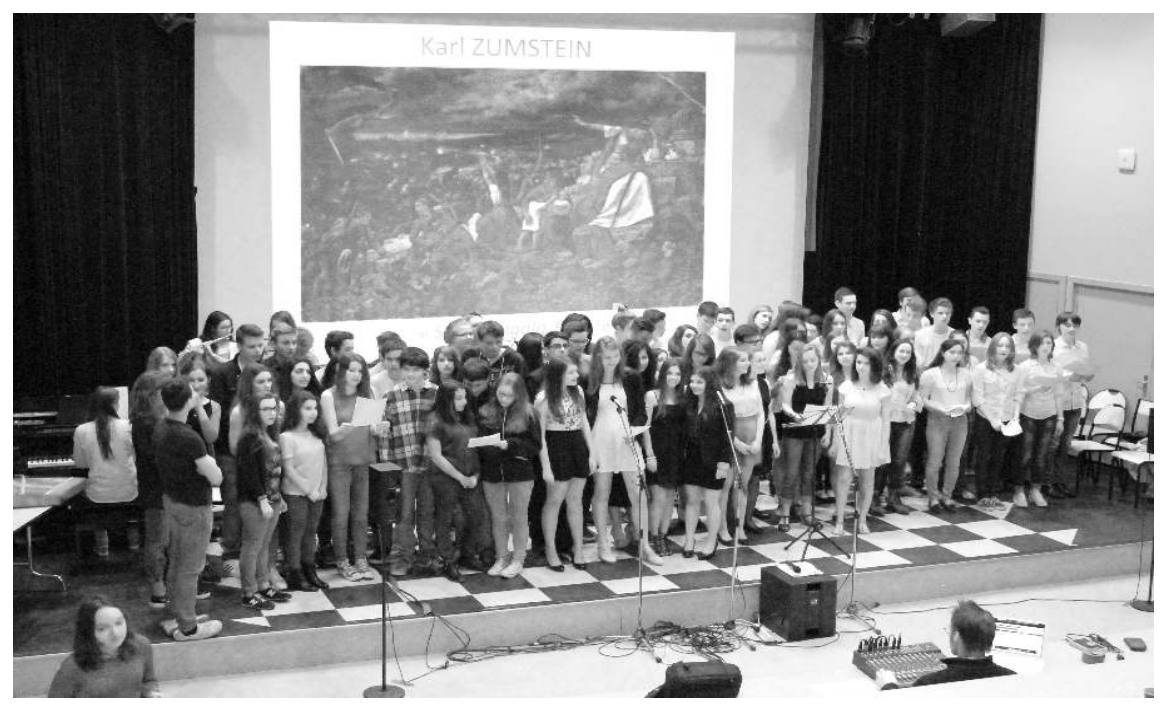

(C) AV, Privatbesitz

Handelt es sich hierbei um ein pädagogisch ausgerichtetes und kein reines Kunstprojekt, so belegt dieses Beispiel dennoch die These des Theaterwissenschaftlers Freddie Rokem, der in Geschichte aufführen schreibt:

„Der Begriff des Aufführens von Geschichte betont die Gegebenheit, dass der Schauspieler, der eine historische Gestalt auf der Bühne aufführt, in gewissem Sinn ebenfalls zu 
einem Zeugen des historischen Ereignisses wird. Als Zeuge muss der Schauspieler nicht unbedingt nach völliger Neutralität oder Objektivität streben, um es den Zuschauern, den ,Umstehenden' im Theater, zu ermöglichen, zu sekundären Zeugen zu werden, zu verstehen und insbesondere sich, ein Urteil [zu] bilden' über die Kräfte, die die Unfälle der Geschichte gestaltet haben. Nicht einmal die Fakten über die Vergangenheit sind völlig ,rein' oder unzweideutig; sie können auf verschiedenen Ebenen angefochten werden, insbesondere wenn sie aus der allmählich zunehmenden Zeitperspektive betrachtet werden “(38)

Der Zugang zu den dramatischen Erlebnissen an der Front verlief in diesem Projekt weniger über die Gestik und das darstellerische Spiel als über die Aneignung der jeweiligen Sprache (z.B. Colmar-Elsässisch im hochdeutschen Lehrkontext), über ihre Verlautbarung und Resonanz im Körper der Sprecher sowie auf der Bühne des Hörsaals (und zwar auf Elsässisch mit französischen und hochdeutschen Untertiteln). $\mathrm{Zu}$ betonen sei ebenfalls, dass der vermeintliche Lesesaal eben gerade durch diese Darbietung zum Auditorium - also Hörsaal - wurde; nicht nur das Publikum (eine kunterbunte Mischung aus Geschwistern, Eltern, Großeltern, Freunden, Mitschülern, Kommilitonen, Kollegen usw.), sondern auch die Performer selbst sind innerhalb der Inszenierung zu Sekundärzeugen des Zeitgeschehens im Brecht'schen Sinne des Wortes geworden. Dieser Eindruck wurde durch auf eine Leinwand projizierte Egodokumente und Live-Vertonungen bestärkt.

Festhalten möchte ich schließlich, dass nicht nur während der Proben im Deutschund Phonetikunterricht eine wahre Gruppendynamik entstand, die es vielen (wenn auch nicht allen) ermöglichte mentale und körperliche Hemmschwellen zu überwinden, sondern auch ein wachsendes Zugehörigkeitsgefühl, das durchaus als transregionale (deutsche Varietäten) und transnationale (deutsch-französische) „Sprachgemeinschaft“ bezeichnet werden darf, die ein kollektives Erinnerungspotential verbirgt. Insofern hat die Aufführung der Kriegsschriften eine vergängliche, aber augenblicklich gegenwärtige Erinnerungsgemeinschaft geschaffen. Auch hier besteht ein Bezug zu Rokems Studie:

„Folglich ist jedes Verfahren, eine Version dessen, was geschehen ist, zu erzählen oder aufzuschreiben, eine Form, Geschichte aufzuführen und jene Vergangenheit zu neuem Leben zu erwecken“(39).

Als „transnational“ kann in diesem Fall die Brückenfunktion der Hochsprache zwischen den Mundarten qualifiziert werden, einerseits zwischen den deutschen Varietäten auf der Textebene (hauptsächlich Varianten des Elsässischen und Badischen), andererseits auf der Ebene des Spiels, also der sprachlichen Umsetzung (hierbei kamen andere Varianten wie z.B. Österreichisch-Deutsch oder Schweizerdeutsch in den Einsatz).

Der künstliche, kodifizierte Rahmen des Spiels und die performative Natur der Sprechhandlungen ermöglichten kurzzeitig einen kollektiven Vergegenwärtigungsprozess einer transregionalen (deutsch-elsässischen), aber auch transnationalen (deutsch-französischen) Vergangenheit, die in einen öffentlichen Bildungsraum

38 Freddie Roкем, Geschichte aufführen. Darstellungen der Vergangenheit im Gegenwartstheater, aus dem Engl. übersetzt von Matthias Naumann (Performing History. Theatrical Representations of the Past in Contemporary Theatre, University of Iowa Press, 2000), Berlin, Neofelis Verlag, 2012, S. 33. 
(Gymnasium und Hochschule) wiederkehren und wachsen durfte. Der dabei geöffnete Erinnerungsraum nahm eine besondere Dimension an, da die territoriale und/ oder sprachliche Zugehörigkeit der elsässischen Einwohner, die seit der Französischen Revolution bekanntlich viermal gewechselt hat, sich auch in ihrem Umgang mit Dialekt und Sprache reflektiert.

Inwiefern zeigen nun die vorherigen Überlegungen, dass das Bühnendeutsch ein transnationales Potential verbirgt oder gar Vektor einer transnationalen deutschensprachigen Öffentlichkeit ist? Tatsächlich ist das viel verwendete Adjektiv nicht eindeutig; es lässt sich etymologisch auf eine gemeinsame Eigenschaft zurückführen, die das Präfix trans- beinhaltet, und die von mindestens zwei Nationen (in unserem Fall Deutschland und Frankreich) geteilt wird. In diesem Sinn unterscheidet es sich von dem Adjektiv „supra-national“, zu Deutsch übernational, das meistens eine politische Macht bezeichnet, die sich über den nationalen Mächten befindet (z.B. die EU). Zudem sollte es nicht mit seinem Zwilling „international“ verwechselt werden, der ein Phänomen (eine Institution) zwischen (inter = entre) Staaten hervorhebt (z.B. die UNO). Transnational ist also etwas, was die territorialen Grenzen von politischen Einheiten überschreitet, ohne dabei die dort geltenden Regeln außer Kraft zu setzen oder zu unterlaufen. Es hat intrinsisch mit Gemeinsamkeit in der Differenz, Einheit in der Vielfalt zu tun und schreibt sich daher in einen Globalisierungsprozess, der landesspezifische Eigenheiten nicht aushebelt.

Durch ihre plurizentrische Natur erscheint mir die deutsche Sprache als ein möglicher Faktor für Transnationalität; sowohl innerhalb Deutschlands, zwischen Bundesländern, als auch außerhalb, wie im elsässisch-französischen Sprachraum. Nach Betrachtung der Entwicklung des Hochdeutschen bzw. der deutschen Standardlautung mag sogar der Gedanke eines „dialektalen Föderalismus“ einleuchten. An dieser Stelle soll eine Gleichung gewagt werden, die die vorigen Erläuterungen bekräftigt:

\begin{tabular}{|l|l|l|}
\hline $\begin{array}{l}\text { 1.a) Langage / } \\
\text { Sprache als Kommunikationssystem }\end{array}$ & $\begin{array}{l}\text { 2.a) La langue nationale / } \\
\text { Landessprache }\end{array}$ & $\begin{array}{l}\text { 3.a) la parole (actes de parole) / } \\
\text { Sprachliche Äußerungen }\end{array}$ \\
\hline $\begin{array}{l}\text { 1.b) régime politique dominant une zone / } \\
\text { Regierungsform; -tendenz }\end{array}$ & $\begin{array}{l}\text { 2.b) Un pays délimité / } \\
\text { Ein begrenztes Land }\end{array}$ & $\begin{array}{l}\text { 3.b) lieu ou endroit particulier / } \\
\text { Ein bestimmter Ort }\end{array}$ \\
\hline
\end{tabular}

In Worte gefasst entspräche der Sprache als Kommunikationssystem (langage) die Struktur einer politischen Regierungsform, während die Landessprache (langue) den Nationalcharakter eines begrenzten Landes nahe käme; schließlich könnte man die einzelnen sprachlichen Äußerungen mit einem bestimmten Ort oder Raum vergleichen (wie Isoglossen sie z.B. eingrenzen).

Eine transnationale Funktion hat also die deutsche Hochsprache in ihrer Standardlautung auf der Bühne: von dort wurde sie nicht nur bezogen, sondern auch verbreitet; man kann sie sozusagen als das „transische“ bzw. "gemeine“ Element bezeichnen, das Mundarten und Varietäten miteinander teilen.

Sie ermöglicht aber auch so etwas wie eine kulturelle Identität, die Grenzen überschreitendes Erinnern und Gedenken ermöglicht. So paradox es klingen mag, bricht 
das „Bühnendeutsch“ Fronten auf, wie es das Wortspiel des von Schülern gewählten Titels für das Projekt typographisch zeigt:

$(\hat{E})$ cri $(\mathrm{t})$ s de guerre $\rightarrow$ Cris de guerre

Im Krieg schrei(b)en $\rightarrow$ Im Krieg schreien

Illustration 4: Plakat der Schüler und Studierenden

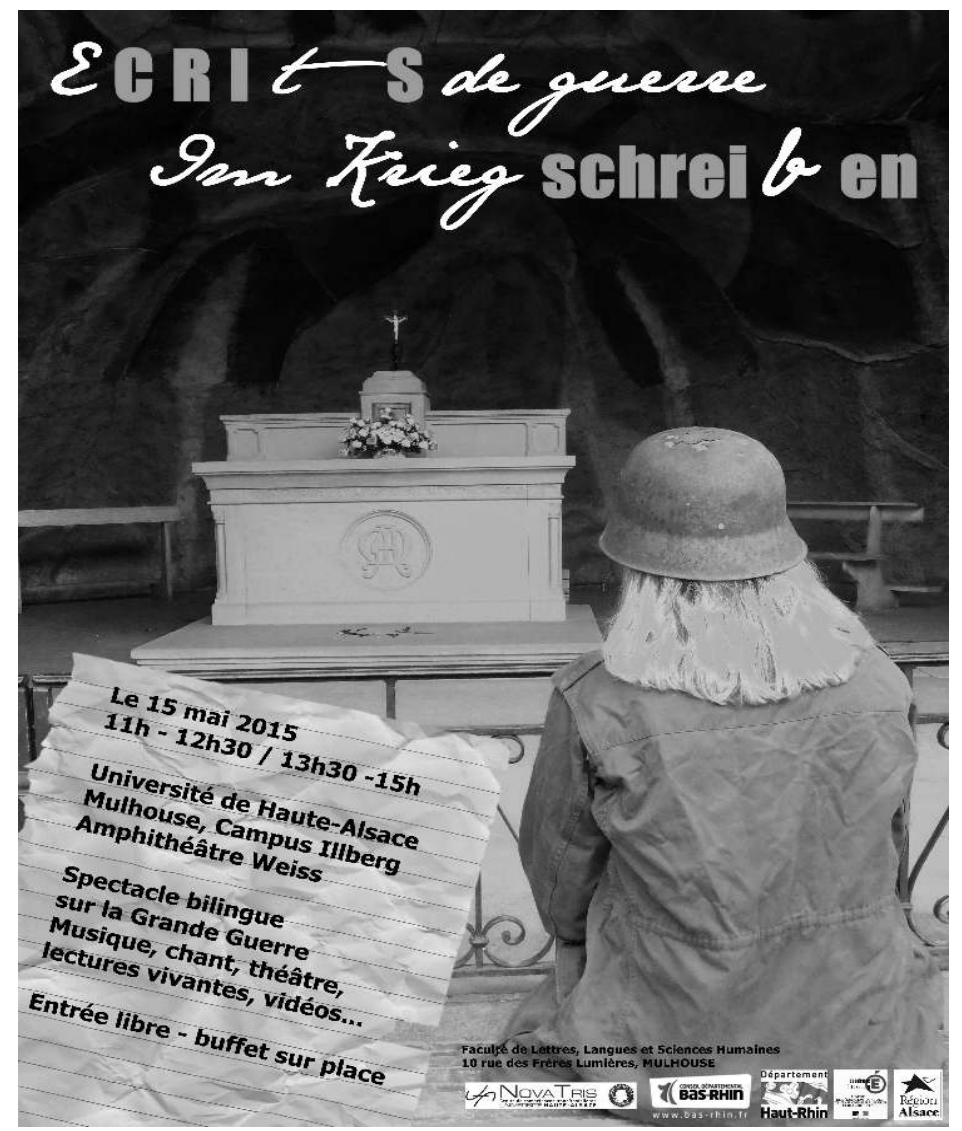

(c) Novatris 2015

Bei dieser Aufführung wurde ein interkultureller und transnationaler Resonanzraum geschaffen, der erhört bzw. gehört werden sollte. Daher seien jene Zeilen zukünftigen Projekten gewidmet, welche die „Trans-Formation“ (40) eines deutschsprachigen öffentlichen Partizipations- und Kooperationsraumes weiterführen möchten.

40 Im wahrsten Sinne des Wortes - geteilte Bildung als Motor des Wandels. 


\section{Zusammenfassung}

Die vorliegende Untersuchung handelt von Identitätsbewegungen, inter-bzw. transnationalem Gedächtnis, schriftlicher und mündlicher Produktion und deren Rezeption im öffentlichen Raum. Dabei wird der Frage nachgegangen, was eigentlich transnational bedeutet, insbesondere in Zusammenhang mit der Öffentlichkeit. Als möglicher transnationaler Raum wird hier der französisch-deutsche Sprachraum betrachtet. Dieser Beitrag hat dabei eine Brückenfunktion, insofern er sowohl den deutschsprachigen als auch einen möglichen transnationalen Raum unter dem Blickwinkel der Performanz im Rahmen der Öffentlichkeit in den Fokus nimmt.

\section{Résumé}

Cette étude se consacre aux mouvements identitaires, à la mémoire internationale, voire transnationale de la production écrite et orale ainsi qu'à sa réception dans l'espace public. Nous nous demanderons en particulier ce que l'adjectif "transnational " peut recouvrir, notamment dans le cadre de l'espace public. L'espace franco-germanophone sera considéré comme étant l'un des espaces transnationaux possibles. Ce faisant, nous tâchons de penser et relier à la fois l'espace germanophone et l'espace transnational sous l'angle de la performance dans l'espace public.

\footnotetext{
Abstract

This study focuses on identity movements, forms of international or transnational memory in written and oral production and its reception in public space. Our main question is about the signification of the adjective "transnational", particularly in the context of public space. The French-German-speaking area will be considered one of the possible transnational spaces. In doing so, we try to think and link together the German-speaking area and the transnational space under the perspective of performance in public space.
} 